\title{
MIB-1 and PC-10 immunostaining for the assessment of proliferative activity in primary acquired melanosis without and with atypia
}

\author{
Itay Chowers, Nelly Livni, Abraham Solomon, Gershom Zajicek, Joseph Frucht-Pery,
} Robert Folberg, Jacob Pe'er

\begin{abstract}
Aims-To compare the proliferative activity of intraepithelial melanocytes in primary acquired melanosis (PAM) without atypia and PAM with atypia by immunohistochemical staining for the Ki-67 antigen and the proliferating cell nuclear antigen (PCNA).

Methods-Formalin fixed, paraffin embedded sections from 35 archival specimens of PAM without atypia $(n=19)$ and with atypia $(n=16)$ were studied by immunostaining with MIB-1 and PC-10 monoclonal antibodies that react with the Ki-67 antigen and PCNA respectively. The results were calculated as the mean number of positive cells per eyepiece grid. All specimens were evaluated by two masked observers, and the interobserver reproducibility was assessed.

Results-The means of the positive cell count in PAM with atypia were significantly higher compared with PAM without atypia for both observers, in both the PC-10 and the MIB-1 stained sections. In a linear least square model that estimated the interobserver and between group variation, the difference of MIB-1 and PC-10 positive cell count between PAM without and with atypia remained highly significant. The difference between the observers was not significant.

Conclusions-Immunostaining with MIB-1 and PC-10 demonstrated that PAM with atypia has higher proliferative activity than PAM without atypia. This method was found to be reproducible between different observers.

(Br f Ophthalmol 1998;82:1316-1319)
\end{abstract}

Clinically, primary acquired melanosis (PAM) is a flat, superficial, and variably brown lesion that is usually unilateral and typically appears in fair complexioned individuals. Several histological conditions account for the clinical presentation of PAM. The condition may result from the deposition of melanin in the conjunctival epithelium without melanocytic hyperplasia or atypia. The condition may also result from melanin deposition that accompanies melanocytic hyperplasia. Histologically, the presence of intraepithelial melanin deposition alone, or with melanocytic hyperplasia without cytological atypia, is designated as PAM without atypia. Such lesions have a negligible association with the subsequent development of malignant melanoma. ${ }^{1-5}$ By contrast, the clinical appearance of a unilateral, flat brown patch in the conjunctiva may be the result of intraepithelial melanocytic hyperplasia with melanocytic atypia, a condition known as PAM with atypia, associated with progression to melanoma in approximately $50 \%$ of patients. ${ }^{1}$

The detection of proliferating cell nuclear antigen (PCNA) and Ki-67 antigen with PC-10 and MIB-1 monoclonal antibodies respectively, was shown to correlate with proliferating activity, histopathological classification, and prognosis in many benign and malignant tumours. ${ }^{6}$ In one study, most lesions classified as PAM with atypia that progressed eventually to conjunctival melanoma were found to be PC-10 positive, while most of the lesions classified as PAM with atypia that did not progress to melanoma were PC-10 negative.

Although the cytological criteria to identify melanocytic atypia in the conjunctiva have been described and illustrated, the histological classification of conjunctival melanocytic lesions remains problematic for many pathologists who do not encounter large numbers of these lesions. This study was designed to study the correlation between the degree of cellular proliferative activity as identified by immunohistochemical demonstration of $\mathrm{Ki}-67$ and PCNA with histological classification of PAM by morphological criteria.

\section{Methods}

Tissue blocks from 35 cases of conjunctival PAM were available for study from the ophthalmic pathology laboratories of the departments of ophthalmology at the Hadassah University Hospital, Jerusalem, and the University of Iowa Hospitals and Clinics, Iowa City, Iowa. All lesions were removed between 1991 and 1996 and had been fixed for 24 hours in $10 \%$ neutral buffered formalin before
Accepted for publication 15 April 1998 

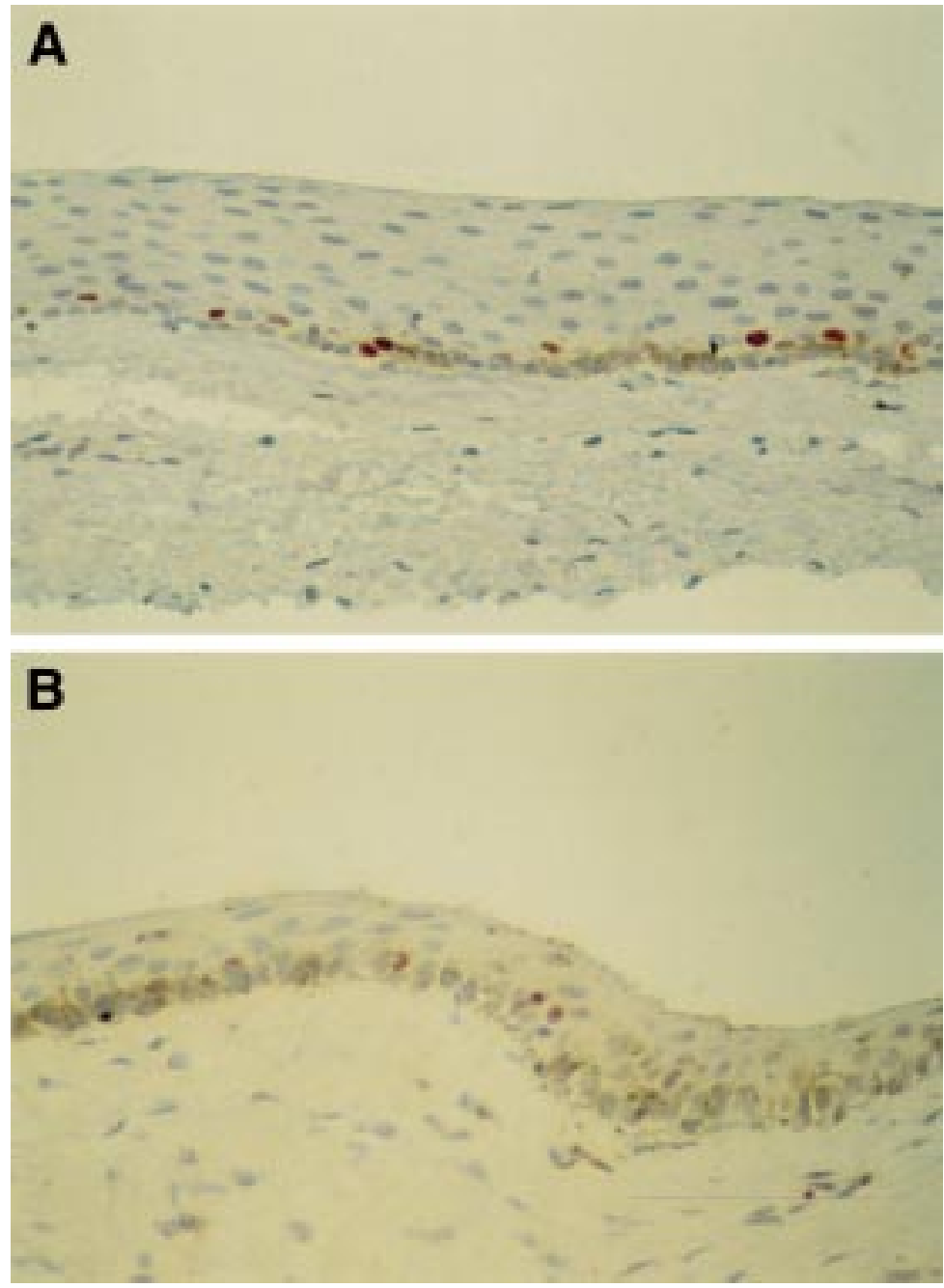

Figure 1 (A) MIB-1 and (B) PC-10 immunostaining of primary acquired melanosis. Positive cells show red nuclear staining in the basilar layer of the epithelium.

original tissue processing. Formalin fixed, paraffin embedded sections were stained with haematoxylin and eosin. The lesions were classified to two groups by ophthalmic pathologists experienced in the histological classification of pigmented conjunctival lesions ( $\mathrm{RF}$ and JP)PAM without atypia $(n=19)$, or PAM with atypia $(n=16)$ according to previously published criteria. ${ }^{1}$ For inclusion into this study, all cases classified as PAM without atypia were required to include a histological component of melanocytic hyperplasia (cases classified as PAM without atypia on the basis of hyperpigmentation but in the absence of melanocytic hyperplasia were excluded from this study).

Paraffin tissue blocks were cut to generate four slides per case, each cut at $6 \mu \mathrm{m}$ thickness. Sections were deparaffinised in xylene and alcohols and placed for 15 minutes in 3\% alcoholic hydrogen peroxide to block endogenous peroxidase. In order to reveal masked antigen, slides were placed in $10 \mathrm{mM}$ citrate buffer $(\mathrm{pH}$ 6.0) and treated in the microwave at $92^{\circ} \mathrm{C}$ for 5 minutes for the PC-10 stained sections and 15 minutes for the MIB-1 stained sections. After removing the container from the microwave and cooling for 15 minutes, slides were placed in phosphate buffered saline (PBS, $\mathrm{pH}$ 7.6).

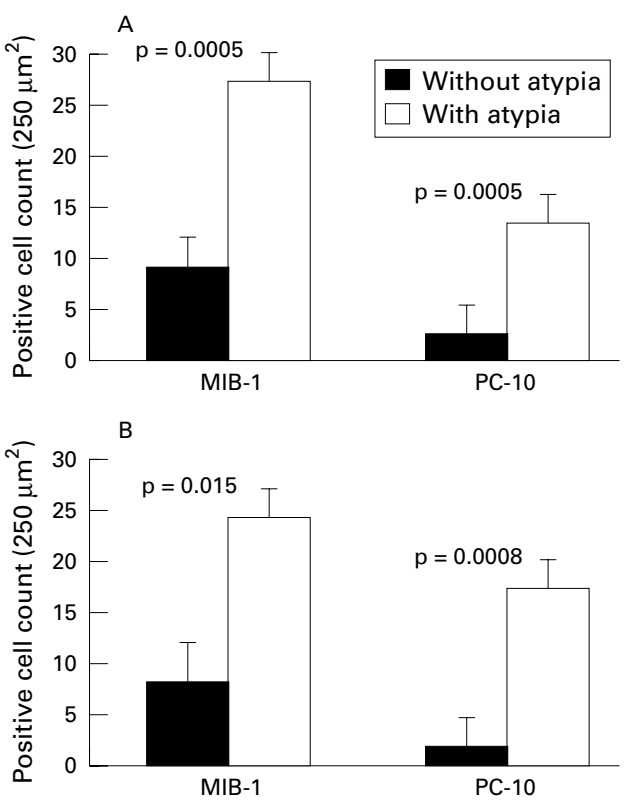

Figure 2 Means and standard errors of $M I B-1$ and $P C-10$ positive cell count for eyepiece grid for $(A)$ observer 1 and $(B)$ observer 2 .

Sections were then treated with bovine serum albumin (BSA) to prevent background staining, and incubated for 1 hour with the primary antibody, PC-10 or MIB-1 (prediluted to 10 $\mu \mathrm{g} / \mathrm{ml}$ and $2 \mu \mathrm{g} / \mathrm{ml}$ respectively, from Zymed Laboratories, Inc, San Francisco, CA, USA), at room temperature in a humidified chamber. Slides were rinsed in PBS for 3-4 minutes and incubated with biotinylated linked antibody for 30 minutes and with labelling reagent peroxidase conjugated streptavidin for 30 minutes (Bio Genex Laboratories, San Ramon, CA, USA). After rinsing, the peroxidase label was demonstrated using 3-amino-9-ethyl carbazole (ACE) for 15 minutes, and counterstained with Mayer haematoxylin. ACE produces a red end product that is soluble in alcohol and is used with an aqueous mounting medium (Kaiser's glycerol gelatin). A negative control was run using the same technique but omitting the primary antibody and adding the streptavidin-biotin complex. In order to increase the number of fields that would be evaluated, two sections from each lesion were stained with PC-10 and two with MIB-1. Immunostaining was assessed in all sections in high power fields $(\times 40)$ using a $10 \times 10$ eyepiece grid $\left(250 \mu \mathrm{m}^{2}\right)$. In each section all the melanocytic cells composing the PAM lesion that contained a distinct positive nuclear staining were regarded as positive. Positive epithelial cells that were not part of the PAM lesion were not counted. Two observers who were masked to the light microscopic classification of the lesion evaluated each section.

Analysis of variance was performed using the JMP statistics software (SAS Institute Inc, Cary, NC, USA). The mean MIB-1 and PC-10 positive cell count for $10 \times 10$ eyepiece grid $\left(250 \mu \mathrm{m}^{2}\right)$ was calculated for both observers in each lesion. To assess the interobserver 
Table 1 Least square means of MIB-1 positive cell count per eyepiece grid $\left(250 \mu^{2}\right)$ estimated with a linear least square model (SD)

\begin{tabular}{ll}
\hline Observer 1 & $17.7(2.5)$ \\
Observer 2 & $16.2(2.8)$ \\
Between observer difference & $\mathrm{p}=0.68$ \\
PAM without atypia & $8.4(2.4)$ \\
PAM with atypia & $25.5(2.8)$ \\
Between groups difference & $\mathrm{p}=0.0001$ \\
\hline
\end{tabular}

Table 2 Least square means of PC-10 positive cell count per eyepiece grid $\left(250 \mu^{2}\right)$, estimated with a linear least square model (SD)

\begin{tabular}{ll}
\hline Observer 1 & $8.0(1.9)$ \\
Observer 2 & $9.3(2.10)$ \\
Between observer difference & $\mathrm{p}=0.62$ \\
PAM without atypia & $2.2(2.0)$ \\
PAM with atypia & $15.1(2.0)$ \\
Between groups difference & $\mathrm{p}=0.0001$ \\
\hline
\end{tabular}

reproducibility the positive cell count of the two observers were fitted by a linear least square model that estimated two differences: interobserver variation and between groups variation.

\section{Results}

Both MIB-1 and PC-10 yielded a distinct red nuclear stain that was easily recognised (Fig 1). The means and standard errors of the MIB-1 and PC-10 positive cell count in PAM without and with atypia for obsevers 1 and 2 are shown in Figure 2. Both observers found that PAM with atypia had a significantly higher MIB-1 and PC-10 positive cell count compared with PAM without atypia. In order to assess the reproducibility of the method, the MIB-1 and PC-10 positive cell counts of the two observers were fitted by linear least square model. In that model PAM with atypia was found to have a significantly higher positive cell count compared with PAM without atypia in both the MIB-1 and PC-10 stained sections. The results of the positive cell count of the two observers were reproducible. The least square means between observers and between groups are presented in Tables 1 and 2 .

\section{Discussion}

Ki-67 monoclonal antibody identifies a 395 kDa nuclear protein that is found in the G1, S, G2, and $M$ phases of the cell cycle. MIB-1 monoclonal antibody recognises $\mathrm{Ki}-67$ antigen, but in contrast with the original $\mathrm{Ki}-67$ antibody, MIB-1 can stain proliferating cells in formalin fixed, paraffin embedded sections. PC-10 is a monoclonal antibody that reacts with the proliferating cell nuclear antigen (PCNA), a $35 \mathrm{kDa}$ acidic nuclear protein that functions as a co-factor for DNA polymerase $\delta$ and is associated with the cell cycle. Before staining with MIB-1 and PC-10, an antigen retrieval by microwave irradiation is needed. Identification of $\mathrm{Ki}-67$ and PC-10 correlated well with the proliferating status of cells as measured by flow cytometry, bromodeoxyuridine, and thymidine labelling and by mitotic counts in many but not all the tumours that have been studied with this technique. ${ }^{68-11}$ For example, in posterior uveal melanoma, there was a direct correlation be- tween measurement of proliferating status by bromodeoxyuridine or flow cytometry and Ki-67 immunostaining. ${ }^{12}$

Ki-67 and PCNA staining correlate also with histopathological features and survival rates in many premalignant and malignant lesions. For instance, in cervical intraepithelial neoplasia (CIN), an epithelial premalignant lesion, MIB-1 staining correlates with the histopathological classification. ${ }^{13}$ Similarly, benign melanocytic tumours of the skin show lower levels of Ki-67 and PCNA than skin melanoma. ${ }^{14} 15$ Few studies have been performed on posterior uveal melanoma. In one study, high Ki-67 but not PCNA levels correlated with larger tumour, tumours of the epithelioid cell type, and shorter survival, ${ }^{9}$ while in another study, high PC-10 counts were associated with death from metastatic uveal melanoma. ${ }^{16} \mathrm{~A}$ third study on 33 choroidal and ciliary body melanomas found no correlation between Ki-67 stain and cellular type, other prognostic factors or survival rates. ${ }^{17}$ Positive identification of PC-10 and Ki-67 in uveal melanomas was also shown to be lower in tumours after brachytherapy. ${ }^{17} 18$

In conjunctival melanoma, higher PC-10 counts were found in late tumours compared with early ones, and these higher levels also correlated with bad prognosis. ${ }^{719}$ In a study of 18 atypical PAM lesions, nine progressed to melanoma and seven of these lesions showed positive PC-10 staining; in contrast, only two of the nine lesions that did not progress to melanoma were PC-10 positive. ${ }^{7}$ However, in these studies the proliferative activity of PAM lesions was not calculated, PAM without atypia was not studied, and Ki-67 levels were not assessed.

We assessed the proliferative activity in PAM with atypia compared with PAM without atypia featuring melanocytic hyperplasia by cytologically normal melanocytes, using PC-10 and MIB-1 monoclonal antibodies. For both markers, each observer found a significantly higher proliferative activity in lesions with atypia. In order to evaluate the reproducibility of the method, we assessed interobserver variability and the significance of the differences between the MIB-1 and PC-10 positive cell count in PAM without and with atypia in a linear least square model. In that model the counts of the two observers were reproducible and the differences between the positive cell count in PAM without and with atypia remained significant.

Although in the present study both stainings showed comparable results, MIB-1 has a few advantages over PC-10. In previous studies, staining of the $\mathrm{Ki}-67$ antigen was found to be a more accurate estimator of the proliferative activity and it was more commonly found to correlate with histopathological grading and prognosis than PC-10 which recognises PCNA..$^{6811}$ The PCNA protein has a relatively long half life so that it remains in the nuclei of cells that are not proliferating but are participating in DNA repair. ${ }^{11}$

The present study was planned to correlate the histological findings in PAM lesions with 
the proliferative activity as assessed by immunostaining. Patients who have conjunctival PAM without atypia seldom progress to melanoma, whereas a significant proportion of patients whose lesions contain cytological atypia do progress to melanoma. For pathologists who require an objective method of separating PAM without atypia from PAM with atypia, immunostaining with proliferation markers such as PC-10 and MIB-1 may be helpful. Further studies are required to determine if proliferation markers are more sensitive than conventional histological classification in identifying patients at higher risk for progression to conjunctival melanoma.

This study was presented in part at the International Symposium on Ocular Tumors, Jerusalem, Israel, 6-10 April, 1997.

Supported in part by an unrestricted grant from Research to Prevent Blindness, Inc (University of Iowa), New York, New York.

1 Folberg R, McLean IW, Zimmerman LE. Primary acquired melanosis of the conjunctiva. Hum Pathol 1985;16:129-35.

2 Folberg R, McLean IW, Zimmerman LE. Conjunctival melanosis and melanoma. Ophthalmology 1984;91:673-8.

3 Folberg R, McLean IW. Primary acquired melanosis and melanoma of the conjunctiva. Hum Pathol 1986;17:652-4.

4 Folberg R. Tumor progression in ocular melanoma. F Invest Dermatol 1993;100:326S-31S.

5 Jakobiec FA, Folberg R, Iwamoto T. Clinicopathologic characteristics of premalignant and malignant melanocytic characteristics of premalignant and malignant melanocytic
lesions of the conjunctiva. Ophthalmology 1989;96:147-66.

$6 \mathrm{Yu}$ CCW, Filipe MI. Update on proliferation-associated antibodies applicable to formalin-fixed paraffin-embedded tissue and their clinical applications. Histochem $\mathcal{F} 1993 ; 25$ $843-53$
7 Seregard S. Cell growth and p53 expression in primary acquired melanosis and conjunctival melanoma. 7 Clin Pathol 1996;49:338-42.

8 Hitchcock CL. Ki-67 staining as a means to simplify analysis of tumor cell proliferation. Am F Clin Pathol 1991;96: $444-6$.

9 Karlsson M, Boeryd B, Carstensen J, et al. Correlation of Ki-67 and PCNA to DNA ploidy, S-phase fraction and survival in uveal melanoma. Eur f Cancer 1996;32A:35762.

10 Hall PA, Levison DA, Woods AL, et al. Proliferating cell nuclear antigen (PCNA) immunolocalization in paraffin section: an index of cell proliferation with evidence of deregulated expression in some neoplasms. I Pathol 1990;162:285-94.

11 Shivji KK, Kenny MK, Wood RD. Proliferating cell nuclear antigen is required for DNA excision repair. Cell 1992;69: 367-74.

12 Bradenstein DS, Char DH, Kaleta-Michaels S, et al. Ki-67 and bromodeoxyuridine labeling of human choroidal melanoma cells. Curr Eye Res 1991;10:479-84.

13 Bulten J, Van Der Laak JAWM, Gemmink JH, et al. MIB-1, a promising marker for the classification of cervical intraepithelial neoplasia. 7 Pathol 1996;178:268-73.

14 Rieger E, Hofmann-Wellenhof R, Soyer P, et al. Comparison of proliferative activity as assessed by proliferating cell nuclear antigen (PCNA) and Ki-67 monoclonal antibodies in melanocytic skin lesions. F Cutan Pathol 1993;20:22936.

15 Fogt F, Vortmeyer AO, Tahan SR. Nuclear organizer regions (AgNOR) and $\mathrm{Ki}-67$ immunoreactivity in cutaneus melanocytic lesions. Am f Dermatopathol 1995;17:12-17.

16 Seregard S, Oskarsson M, Spangberg B. PC-10 as a predictor of prognosis after antigen retrieval in posterior uveal
melanoma. Invest Ophthalmol Vis Sci 1996;37:1451-8.

17 Mooy CM, de Jong PTVM, Van Der Kwast TH, et al. Ki-67 immunostaining in uveal melanoma. Ophthalmology 1990; 97:1275-80.

18 Pe'er J, Gnessin H, Shargal Y, et al. PC-10 immunostaining of proliferating cell nuclear antigen in posterior uveal melanoma. Ophthalmology 1994;101:56-62.

19 Seregard S. Cell proliferation as a prognostic indicator in conjunctival malignant melanoma. Am f Ophthalmol 1993; 116:93-7 